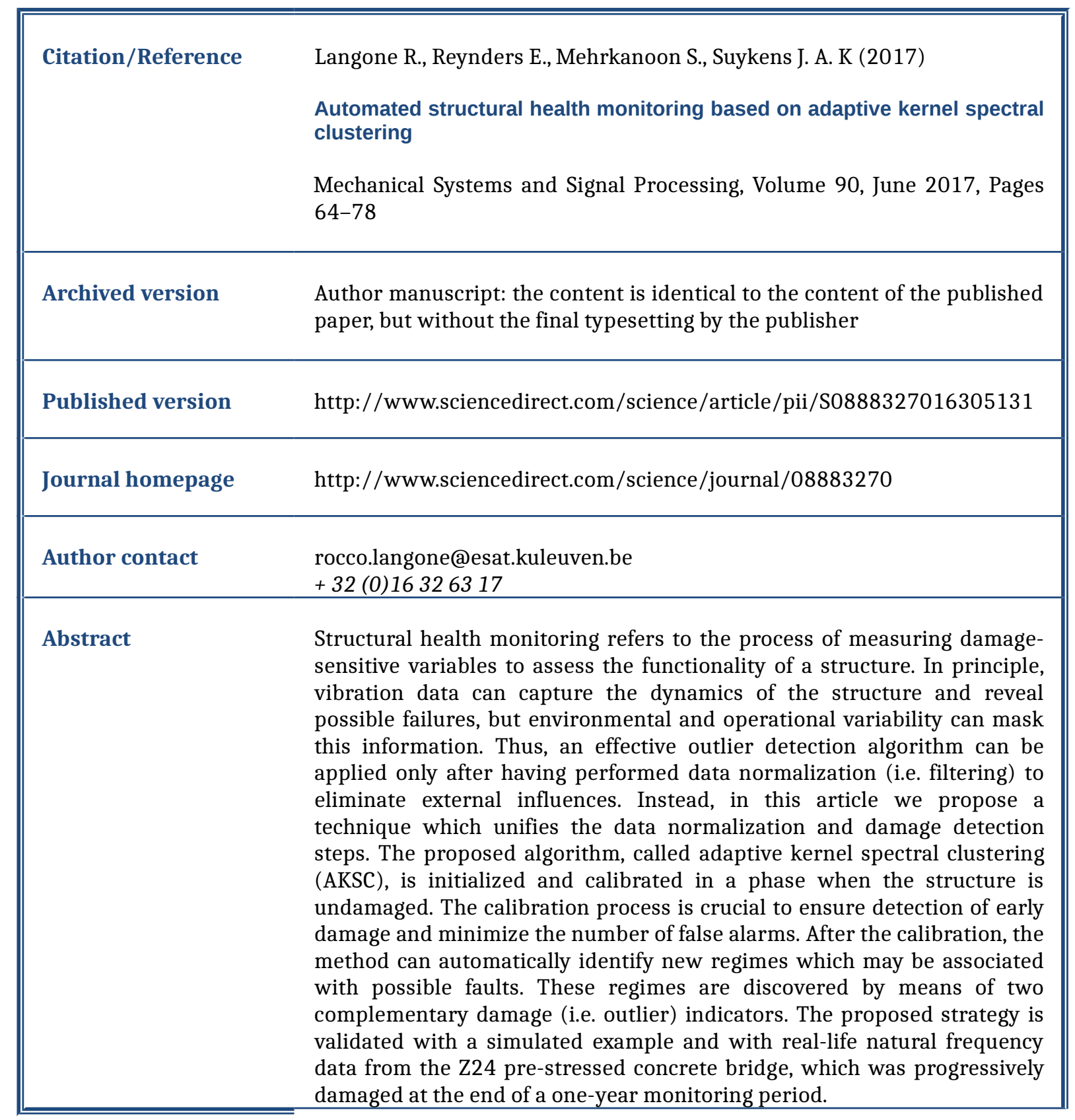




\title{
Automated structural health monitoring based on adaptive kernel spectral clustering
}

\author{
Rocco Langone $^{1 \mathrm{a}}$, Edwin Reynders ${ }^{\mathrm{b}}$, Siamak Mehrkanoon ${ }^{\mathrm{a}}$, Johan A. K. Suykens ${ }^{\mathrm{a}}$ \\ ${ }^{a}$ KU Leuven, Stadius Centre for Dynamical Systems, Signal Processing and Data Analytics - ESAT, \\ Kasteelpark Arenberg 10, B-3001 Leuven, Belgium. \\ ${ }^{b}$ KU Leuven, Department of Civil Engineering, Kasteelpark Arenberg 40, B-3001 Leuven, Belgium.
}

\begin{abstract}
Structural health monitoring refers to the process of measuring damage-sensitive variables to assess the functionality of a structure. In principle, vibration data can capture the dynamics of the structure and reveal possible failures, but environmental and operational variability can mask this information. Thus, an effective outlier detection algorithm can be applied only after having performed data normalization (i. e. filtering) to eliminate external influences. Instead, in this article we propose a technique which unifies the data normalization and damage detection steps. The proposed algorithm, called adaptive kernel spectral clustering (AKSC), is initialized and calibrated in a phase when the structure is undamaged. The calibration process is crucial to ensure detection of early damage and minimize the number of false alarms. After the calibration, the method can automatically identify new regimes which may be associated with possible faults. These regimes are discovered by means of two complementary damage (i. e. outlier) indicators. The proposed strategy is validated with a simulated example and with real-life natural frequency data from the Z24 pre-stressed concrete bridge, which was progressively damaged at the end of a one-year monitoring period.
\end{abstract}

Keywords: Structural health monitoring, data normalization, novelty detection, bridge engineering, adaptive kernel spectral clustering.

\section{Introduction}

Structural health monitoring (SHM) is regarded as the main tool to implement a damage identification strategy for any engineering structure [1]. SHM techniques may follow different approaches according to how sensor data are used for the decisionmaking [2]. In this paper we focus on methods using data mining for extracting sensitive information from time-series, such as vibration response data produced by accelerations or strains. Although sensor data such as accelerations or strains can be employed directly as damage-sensitive features for SHM, it is common practice to convert them first into modal characteristics such as natural frequencies and (strain) mode shapes

\footnotetext{
${ }^{1}$ Corresponding author: rocco.langone@esat.kuleuven.be
} 
[3]. This has two major advantages: (1) while the directly measured signals depend on the excitation, this is not the case for the modal characteristics, who only depend on structural properties, and (2) the amount of data is heavily reduced without losing essential information about the structure.

Data-driven methods permit to overcome the difficulty of building-up complex physical models of the system. However, in order to use sensor data to perform structural health monitoring with reasonable success, environmental conditions must be taken into account. This is due to the fact that in the vibration signals the changes in structural performance are entangled with regular changes in temperature, relative humidity, operational loading. If not accounted for, these external influences can prevent to correctly identify failures. For instance, in [4] the authors showed that a nonlinear model is necessary to filter out the operational variability, because the influence of the environment on the observed damage-sensitive features is physically very complex. In particular, the features extracted using kernel principal component analysis (kernel PCA) were found to have a better discriminative power compared to (linear) PCA in the analysis of the Z24 bridge dataset. In [5] robust regression analysis has been used to discriminate between benign variation in the environmental and operating conditions and structural damage in case of the Z24 and Tamar bridges. The authors of [6] have devised a two-step procedure based on a Gaussian process model which allows to first separate the environmental and operational effects from sensor fault and structural damage and afterwards to discriminate between the latter two conditions. In [7] the development of a stochastic framework that efficiently fuses operational response data with external influencing agents for representing structural behavior in its complete operational spectrum is reviewed.

Within the structural damage detection methods, one-class outlier analysis has been used for a long time and is considered among the most popular class of techniques. In [8] a conceptually simple approach based on Mahalanobis squared distance (MSD) is devised: an observation is labelled as outlier if its discordancy value is greater than a threshold, which is determined using a Monte Carlo method. Sohn et al. [9] use a three-step procedure validated on simulated data. First, an autoregressive with exogenous inputs (ARX) model is developed to extract damage-sensitive features, then an autoassociative neural network (AANN) is employed for data normalization, and finally a sequential probability ratio test is performed on the normalized features to automatically infer the damage state of the system. A similar approach is followed in [10] in the study of the Alamosa Canyon Bridge dataset, where the performance of four different techniques (namely AANN, MSD, factor analysis and singular value decomposition) is assessed in terms of receiver operating characteristics (ROC) curves. The main difference with [9] is that each model performs data normalization and at the same time produces a scalar output that is used as a damage indicator. In [11] one-class support vector machine was successfully used to detect faults in rotors with high precision. Deraemaeker et al. [12] introduce two types of features, namely eigenproperties of the structure and peak indicators. These features are then fed to a factor analysis model to treat the effects of the environment, and damage is detected using the multivariate Shewhart-TT control charts. Also in [13] control charts are used for damage identification in an arch bridge. Moreover, regression models complemented with PCA are employed beforehand to minimize the effects of environmental and operational factors 
on the bridge natural frequencies.

Although much less popular than one-class methods, cluster analysis [14] has also been explored as a possible tool to perform structural health monitoring. In [15] the k-means algorithm is applied to features which have been previously extracted using time-reversal acoustics. Here the number of clusters is fixed to two by assuming that there are only two distinct groups of data points, related to undamaged and damage condition. In the approach by Kesevan et al. [16], first some damage sensitive features based on the energies of the Haar and Morlet wavelet transforms of the vibration signal are extracted. Then PCA is applied to create a database of normalized baseline signals and finally $\mathrm{k}$-means is employed for the decision-making. In particular, the gap statistic is used to determine the optimal number of clusters, and in case more than one cluster is found while comparing the damage sensitive feature of the closest baseline signal and signal being analysed, then a damage is detected. Palomino et al. [17] compare C-means and Gustafson-Kessel fuzzy cluster algorithms in their ability to implement impedance-based SHM, which utilizes the electromechanical coupling property of piezoelectric materials as non-destructive evaluation method [18]. Notably, a riveted aluminium beam equipped with piezoelectric sensors was used as test case. Both clustering algorithms were able to correctly identify from the impedance signals two $^{2}$ types of damage induced on purpose, i.e. crack and rivet loss. Fuzzy C-means is used also in [19] to cluster Heavy Weight Deflectometer data collected at an airport pavement in Italy, in order to evaluate its structural behaviour. In [20] the identification of structural changes in the five-span suspended Samora Machel Bridge is performed using the dynamic cloud clustering algorithm [21].

In this article an adaptive methodology based on an iterative spectral clustering method is presented. Spectral clustering techniques make use of the eigenvectors of the so called Laplacian matrix to map the original data into a lower dimensional space, where clustering is performed [22, 23, 24]. Recently, a kernel spectral clustering (KSC) method has been introduced [25], which casts spectral clustering in a kernel-based learning framework. In the KSC setting, it is possible to choose the number of clusters and the kernel parameters by means of a systematic model selection procedure. Furthermore, KSC allows to predict the cluster memberships for out-of-sample data in a straightforward way. This out-of-sample extension property is exploited in the proposed approach to update the initial clustering model.

The proposed method, named adaptive kernel spectral clustering (AKSC), has several advantages compared to existing techniques:

- the clustering model is able to adapt itself to a changing environment. The number of clusters (related to both damaged and undamaged conditions) can change over time after the initialization and calibration period, allowing a more accurate detection of faults. This ability to model the structural changes over time by detecting new regimes ${ }^{3}$ allows to unify the data normalization and damage detection steps in a single procedure.

\footnotetext{
${ }^{2}$ The number of clusters has been fixed to 3 in order to distinguish between the undamaged and the two different damaged conditions.

${ }^{3} \mathrm{New}$ regimes relate to merging of existing clusters or creation of new clusters.
} 
- a small number of data points is needed for constructing an initial clustering model

- in the initialization and the calibration periods the algorithm hyper-parameters are determined in a rigorous manner by means of a systematic tuning procedure. In the initial stage optimal choices for the kernel bandwidth $\sigma$ and the number of clusters $k$ are made by means of cross-validation in conjunction with a gridsearch procedure. A KSC model is built for every grid-point (defined by a certain $k, \sigma$ pair) on a training set, cluster memberships for out-of-sample points in a separated validation set are obtained, and the related cluster quality is computed. In particular, the average membership strength (AMS) criterion [26] is used as performance function, which (roughly speaking) selects the KSC parameters that maximize the separation between the clusters. Finally, the model reaching the highest AMS score is selected (see Figure 4). In the calibration phase, an online model selection scheme is devised to adapt the initial $k, \sigma$ pair and meet userdefined fault tolerance specifications.

- two different damage indicators are introduced. They are validated on both simulated and experimental data, and are shown to allow the detection of suspicious structural behaviour upon their occurrence.

The remainder of this article is organized as follows. In Section 2 the new approach for real-time structural health monitoring is introduced. Section 3.1 concerns the validation of this procedure by means of a synthetic example. In Section 3.2 a discussion of the experimental results obtained on the Z24 bridge benchmark is given. Moreover, a comparison with the fuzzy C-means algorithm is performed. Finally, Section 4 concludes the paper and proposes future research directions.

\section{Proposed damage detection strategy}

In this Section an adaptive strategy for the automatic structural assessment in realtime is introduced. The proposed approach exploits the incremental updating mechanism proposed in [27] to build a reliable and realistic fault detection procedure. In the new method, that is named adaptive kernel spectral clustering (AKSC), the initialization phase is followed by a calibration period where a desired clustering model is selected. As a consequence, a model which does not produce more false alarms than an accepted tolerance threshold and at the same time is sensitive enough to recognize possible failures, is obtained. Furthermore, two different outlier indicators are provided. Before going into the technical details and to facilitate the next reading, in Figure 1 a flowchart of the proposed strategy at the top and, at the bottom side, the output obtained by running the AKSC algorithm on the Z24 bridge dataset described in Section 3.2 are shown.

\subsection{Initialization}

In the first monitoring period an initial clustering model is built-up. In particular, a kernel spectral clustering (KSC [25]) algorithm is used to cluster the data. The KSC 

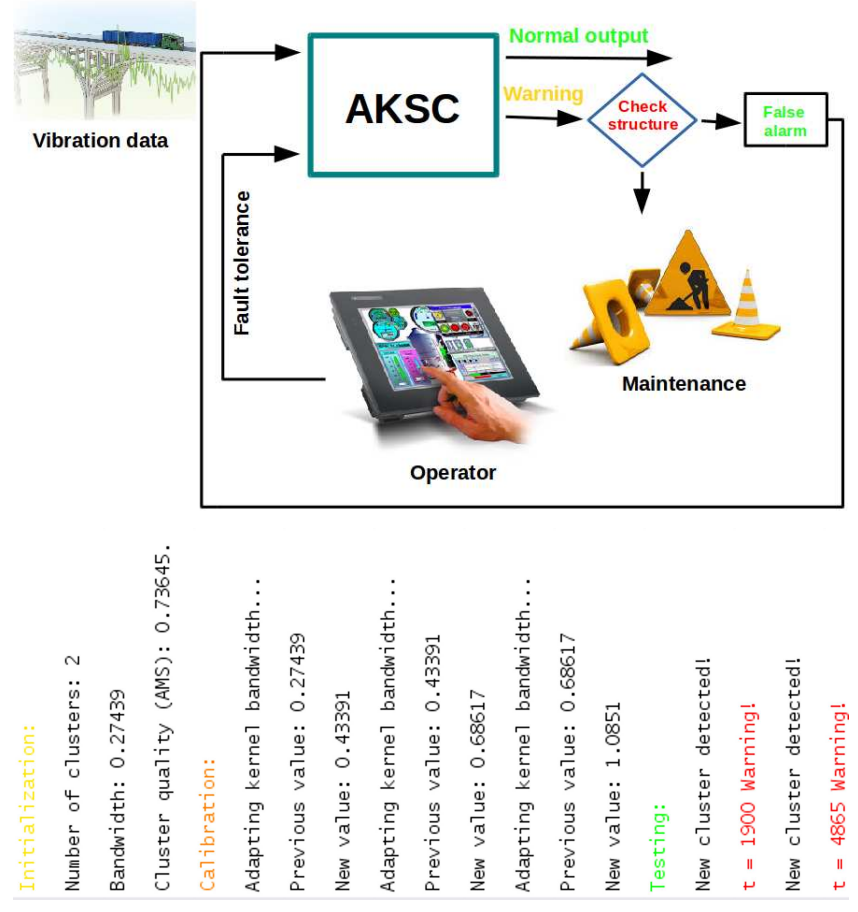

Figure 1: Proposed strategy. (Top) Illustrative picture of the proposed AKSC-based structural health monitoring approach. (Bottom) Output snippet when running the AKSC algorithm from Matlab2015a. In this case the fault tolerance threshold has been set as tol $l_{\text {new }}=1$ at the beginning of the calibration period. Furthermore, in the test stage both warnings have been ignored in order to show the clustering evolution on the entire dataset (see Figure 9 for more details).

method allows to discover complex nonlinear cluster boundaries because the original data are mapped into a new space called feature space, where groups of similar points can be detected more easily. In particular, according to the theory of kernel methods [28], a nonlinear model in the input space can be obtained by (1) mapping the original data to the feature space and (2) designing a linear model in this new space. This concept is illustrated in Figure 2.

For a given set of training data $\mathcal{D}_{\mathrm{tr}}=\left\{\mathbf{x}_{i}\right\}_{i=1}^{N_{\text {tr }}}$, with $\mathbf{x}_{i} \in \mathbb{R}^{d}$, that we want to group into $k$ clusters, the KSC objective can be formulated as follows:

$$
\begin{array}{ll}
\min _{w^{(l)}, e^{(l)}, b_{l}} & \frac{1}{2} \sum_{l=1}^{k-1} w^{(l)^{T}} w^{(l)}-\frac{1}{2} \sum_{l=1}^{k-1} \gamma_{l} e^{(l)^{T}} D^{-1} e^{(l)} \\
\text { subject to } & e^{(l)}=\Phi w^{(l)}+b_{l} 1_{N_{\mathrm{Tr}}} .
\end{array}
$$

The symbols have the following meaning:

- $w^{(l)} \in \mathbb{R}^{d_{h}}$ and the bias term $b_{l}$ represent the parameters of the model, which is represented by an hyper-plane

- $e^{(l)} \in \mathbb{R}^{N_{\text {tr }}}$ are the projections of the $N_{\text {tr }}$ datapoints in the space spanned by the vectors $w^{(1)}, \ldots, w^{(k-1)}$ 

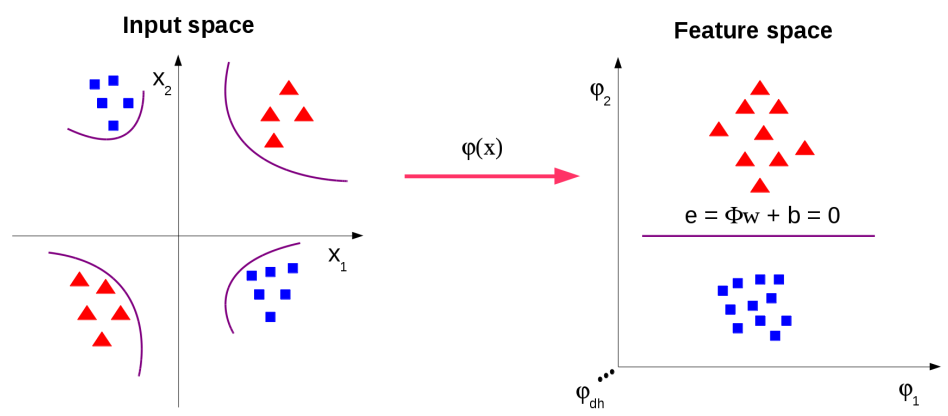

Figure 2: Clustering in the feature space. Mapping of the input data to a high dimensional feature space (of dimension $d_{h}$ ) where a linear separation is made, which is related to a nonlinear clustering boundary in the input space.

- $\Phi=\left[\varphi\left(\mathbf{x}_{1}\right)^{T} ; \ldots ; \varphi\left(\mathbf{x}_{N_{\mathrm{tr}}}\right)^{T}\right]$ is the feature matrix, where $\varphi: \mathbb{R}^{d} \rightarrow \mathbb{R}^{d_{h}}$ denotes the mapping to a high-dimensional feature space

- the matrix $D$ is referred to as the degree matrix.

Objective (1) casts the multi-way KSC model as a weighted kernel PCA formulation [29], with the weighting matrix being equal to the inverse of the degree matrix $D^{-1}$. This choice leads to the dual problem (3), which is related to spectral clustering. Optimization problem (1) can be interpreted as finding a new coordinate system $w^{(1)}, \ldots, w^{(k-1)}$ such that the weighted variances of the projections $e^{(l)}, l=$ $1, \ldots, k-1$ in this new basis, i. e. $e^{(l)^{T}} D^{-1} e^{(l)}$, are maximized (in this sense KSC is related to kernel principal component analysis). If the reader refers to Figure 2, this is equivalent to saying that the squared distances to the cluster boundary $e^{(l)}=0$ must be as large as possible (to have a better separation between the clusters). Furthermore, the contextual minimization of the squared norm of the vector $w^{(l)}$ is desired, in order to trade-off the model complexity expressed by $w^{(l)}$ with the correct representation of the training data. The variables $\gamma_{l}$ are regularization constants. From a practical point of view, since KSC represents at the same time a kernel PCA model and a clustering algorithm, it allows us to unify data normalization and damage detection in a single approach.

In principle, specifying explicitly the feature map $\varphi(\cdot)$ can require a big effort in terms of feature engineering. In order to avoid this complex task, it is convenient to derive the dual formulation corresponding to the primal problem (1). By doing so, as will be clear soon, one can use the so called kernel trick to operate in the feature space without ever computing the coordinates of the data in that space. Instead, one needs to simply calculate the inner products between the images of all pairs of data in the feature space, i.e. $\varphi\left(\mathbf{x}_{i}\right)^{T} \varphi\left(\mathbf{x}_{j}\right)$.

The inner products represent the similarity between each pair of datapoints, which is defined by a specific kernel function. A popular kernel function, that will be also employed throughout this paper, is the radial basis function (RBF) kernel $K$ defined as $K\left(\mathbf{x}_{i}, \mathbf{x}_{j}\right)=\exp \left(-\frac{\left.\left\|\mathbf{x}_{i}-\mathbf{x}_{j}\right\|_{2}^{2}\right)}{2 \sigma^{2}}\right)$. The parameter $\sigma$ is usually referred to as the bandwidth, and controls the complexity of the nonlinear mapping implicitly defined by the 
kernel.

It can be shown [25] that, under certain conditions for the nonlinear mapping $\varphi(\cdot)$, the variables $e^{(l)}$ that appear in eq. (1) can be obtained as:

$$
e_{i}^{(l)}=\sum_{j=1}^{N_{\mathrm{tr}}} \alpha_{j}^{(l)} K\left(\mathbf{x}_{j}, \mathbf{x}_{i}\right)+b_{l}, l=1, \ldots, k-1
$$

where index $i$ refers to the $i$-th datapoint. The bias term $b_{l}$ can be calculated as $b_{l}=$ $-\frac{1}{\mathbf{1}_{N_{\mathrm{tr}}}^{T} D^{-1} \mathbf{1}_{N_{\mathrm{tr}}}} \mathbf{1}_{N_{\mathrm{tr}}}^{T} D^{-1} \Omega \alpha^{(l)}$, being $\mathbf{1}_{N_{\mathrm{tr}}}$ a vector of ones. The $\alpha$ terms follow from the solution of the following eigenvalue problem:

$$
D^{-1} M_{D} \Omega A=A \Lambda
$$

where

- $A$ is a matrix of dimension $N_{\text {tr }} \times(k-1)$ whose columns are the $k-1$ eigenvectors corresponding to the largest eigenvalues, i. e. $A=\left[\alpha^{(1)}, \ldots, \alpha^{(l)}, \ldots, \alpha^{k-1}\right]$, with $\alpha^{(l)} \in \mathbb{R}^{N_{\text {tr }}}$

- $\Lambda$ is the diagonal matrix whose diagonal elements are the eigenvalues $\lambda_{1}, \ldots, \lambda_{k-1}$

- $\Omega$ is the kernel matrix with $i j$-th entry $\Omega_{i j}=K\left(\mathbf{x}_{i}, \mathbf{x}_{j}\right)=\varphi\left(\mathbf{x}_{i}\right)^{T} \varphi\left(\mathbf{x}_{j}\right)$

- as anticipated earlier, $D$ is the degree matrix, which is diagonal with positive elements $D_{i i}=\sum_{j} \Omega_{i j}$

- $K: \mathbb{R}^{d} \times \mathbb{R}^{d} \rightarrow \mathbb{R}$ is the kernel function, that is a function which outputs a high value when evaluated on similar data points and a low value for dissimilar inputs

- $\varphi: \mathbb{R}^{d} \rightarrow \mathbb{R}^{d_{h}}$ denotes the mapping to a high-dimensional feature space, as before

- $M_{D}$ is a centering matrix defined as $M_{D}=I_{N_{\mathrm{tr}}}-\frac{1}{1_{N_{\mathrm{tr}}}^{T} D^{-1} 1_{N_{\mathrm{tr}}}} 1_{N_{\mathrm{tr}}} 1_{N_{\mathrm{tr}}}^{T} D^{-1}$.

Notice that by solving (3) instead of (1), the problem of specifying the nonlinear mapping $\varphi(\cdot)$ is circumvented, as only inner products (i.e. $\Omega_{i j}=K\left(\mathbf{x}_{i}, \mathbf{x}_{j}\right)=$ $\varphi\left(\mathbf{x}_{i}\right)^{T} \varphi\left(\mathbf{x}_{j}\right)$ ) appear in equation (3).

The cluster assignment can be obtained by applying the sign function to $\mathbf{e}_{i}$, which is then referred also as clustering score or latent variable. The binarization is straightforward because the bias term $b_{l}$ has the effect of centering $e^{(l)}$ around zero.

After binarizing the clustering scores of all the training points as $\operatorname{sign}\left(\mathbf{e}_{i}\right)$, a codebook with the most frequent binary indicators is formed. For example in case of three clusters $(k=3, l=1,2)$ it may happen that the most occurring code-words are given by the set $\mathcal{C}_{B}=\{[11],[-11],[-1-1]\}$. Thus, the codebook $\mathcal{C}_{B}$ contains the cluster prototypes, and the cluster membership for each training point are obtained via an error correcting output codes (ECOC) decoding procedure. The ECOC scheme works as follows:

- for a given training point $\mathbf{x}_{i}$, compute its projection $\mathbf{e}_{i}=\left[e_{i}^{(1)}, e_{i}^{(2)}\right]$ as in eq. (2) 
- binarize $\mathbf{e}_{i}$ as $\operatorname{sign}\left(\mathbf{e}_{i}\right)$

- suppose that $\operatorname{sign}\left(\mathbf{e}_{i}\right)=\left[\begin{array}{ll}1 & 1\end{array}\right]$, then assign $\mathbf{x}_{i}$ to cluster 1 (i. e. the closest prototype in the codebook $\mathcal{C}_{B}$ in terms of Hamming distance).

In the cases where $\operatorname{sign}\left(\mathbf{e}_{i}\right)$ has the same Hamming distance to more than one prototype, then $\mathbf{x}_{i}$ is assigned to the cluster whose mean value is closer to $\mathbf{e}_{i}$ in terms of Euclidean distance. In our example, this would occur when $\operatorname{sign}\left(\mathbf{e}_{i}\right)=[1-1]$, whose Hamming distance from [1 1$]$ and $[-1-1]$ is the same. In Figure 3 an illustrative picture of the ECOC coding scheme is shown.
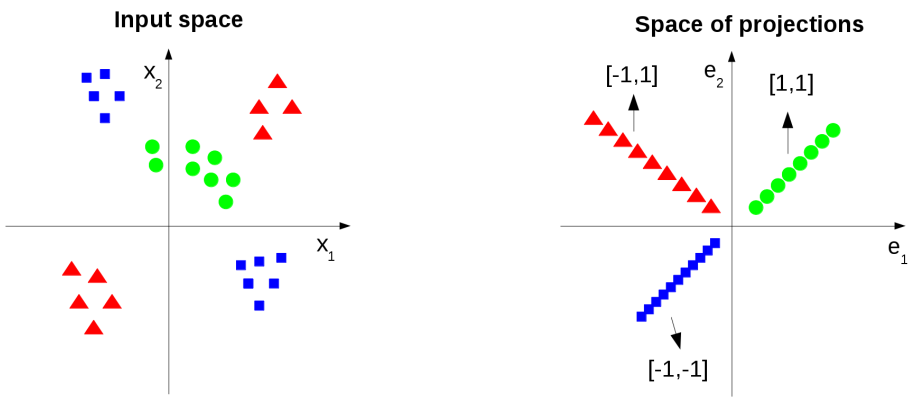

Figure 3: ECOC coding procedure. The orthant in which the clustering scores $e^{(l)}$ lie determines their sign pattern and the corresponding cluster prototype.

Since KSC is cast in a kernel-based optimization setting, it is important to perform model selection to choose the kernel parameters and discover the number of clusters present in the data. For instance, in case of the RBF kernel, a bad choice of its bandwidth parameter $\sigma$ can compromise the quality of the final clustering results.

Another advantage provided by the KSC technique is its out-of-sample property. A new (test) point, say $\mathbf{x}_{i, \text { test }}$, can be clustered in a straightforward way by following two simple steps:

- the test clustering score is computed as $\mathbf{e}_{i, \text { test }}=\left[e_{i, \text { test }}^{(1)}, \ldots, e_{i, \text { test }}^{(k-1)}\right]$, with $e_{i, \text { test }}^{(l)}=$ $\sum_{j=1}^{N_{\mathrm{tr}}} \alpha_{j}^{(l)} K\left(\mathbf{x}_{j}, \mathbf{x}_{i, \text { test }}\right)+b_{l}$

- after calculating $\operatorname{sign}\left(\mathbf{e}_{i, \text { test }}\right)$, assign point $\mathbf{x}_{i, \text { test }}$ to the closest cluster prototype present in the codebook $\mathcal{C}_{B}$, using the ECOC decoding scheme mentioned earlier.

In order to facilitate the reader in understanding the working mechanism of the KSC algorithm, in Figure 4 an example of clustering obtained on a toy dataset is depicted.

\subsection{Calibration}

Once an initial grouping of the data at hand has been obtained, the clustering model needs to be updated in order to cope with the future data evolution. For this purpose, the cluster centroids in the eigenspace ${ }^{4} C_{\alpha}^{1}, \ldots, C_{\alpha}^{k}$ are computed, and a new cluster

${ }^{4}$ We remind that the eigenspace is the space spanned by the vectors $\alpha^{(1)}, \ldots, \alpha^{(k-1)}$. 

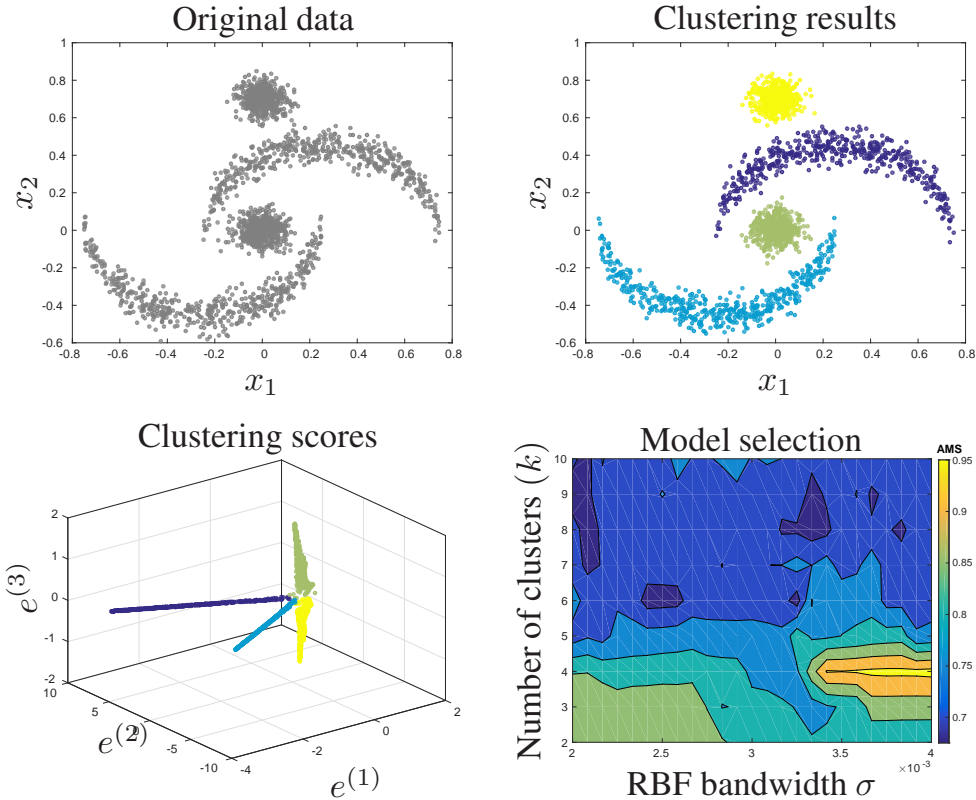

Figure 4: Clustering produced by the KSC algorithm on a toy dataset. (Top left) Original dataset consisting of 4 clusters. (Top right) clustered data. (Bottom left) Points represented in the space spanned by the score variables $e^{(1)}, e^{(2)}, e^{(3)}$. (Bottom right) Model selection plot used to determine the number of clusters $k$ and the bandwidth parameter $\sigma$, obtained by using the average membership strength (AMS) model selection criterion [26] mentioned in the Introduction. In this case the tuned parameters are $k=4$ and $\sigma=3.8 \cdot 10^{-3}$. 
assignment rule is devised. In particular, for every new data-point $\mathbf{x}_{i \text {,new }}$ its coordinates in the eigenspace $\alpha_{i, \text { new }}=\left[\alpha_{i \text {,new }}^{(1)}, \ldots, \alpha_{i, \text { new }}^{(k-1)}\right]$ can be calculated using the following equation [30]:

$$
\alpha_{i, \text { new }}^{(l)}=\frac{e_{i, \text { new }}^{(l)}}{\lambda_{l} \operatorname{deg}\left(\mathbf{x}_{i, \text { new }}\right)}
$$

with $\operatorname{deg}\left(\mathbf{x}_{i, \text { new }}\right)=\sum_{j=1}^{N_{\text {tr }}} \alpha_{j}^{(l)} K\left(\mathbf{x}_{j}, \mathbf{x}_{i, \text { new }}\right)$. The cluster membership for $\mathbf{x}_{i \text {, new }}$ can be computed as measured by the Euclidean distance from the cluster centroids, similarly to k-means clustering 5 . In Figure 5 this alternative assignment rule is illustrated, where the same toy example employed in Figure 4 has been used.
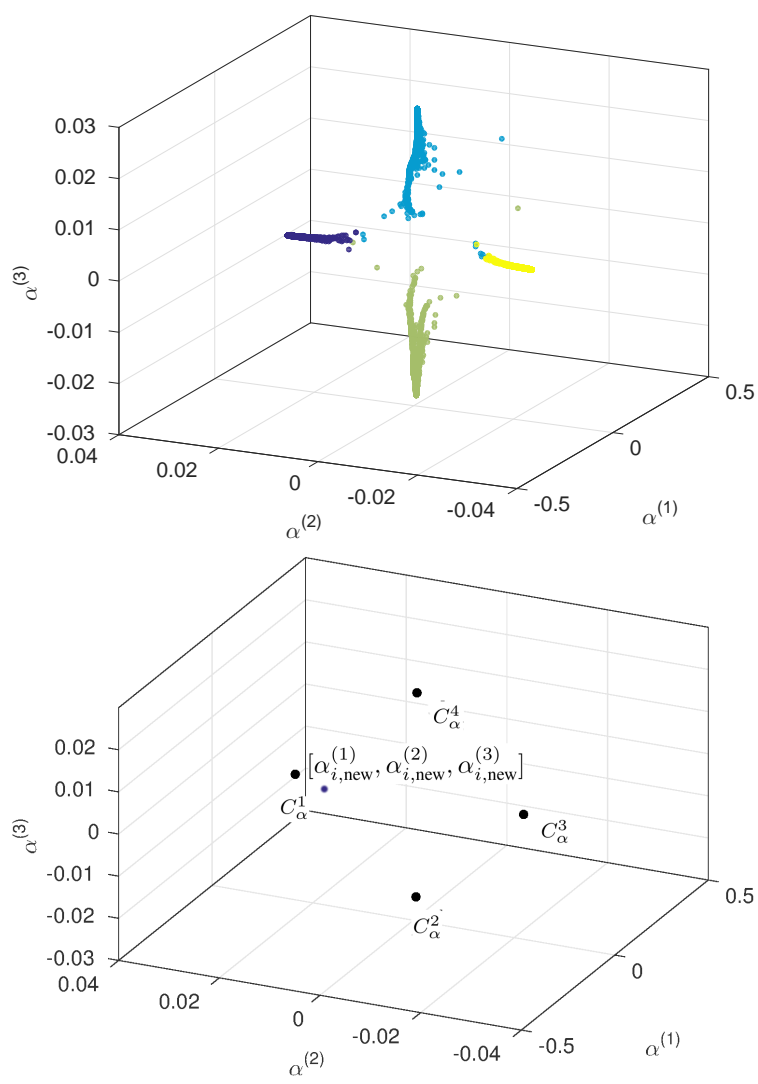

Figure 5: Illustration of the cluster membership assignment rule based on the distance from centers in the eigenspace. (Top) Representation of the toy dataset in the (training) eigenspace spanned by $\alpha^{(1)}, \alpha^{(2)}, \alpha^{(3)}$. (Bottom) A new (test) point is assigned to the closest cluster centroid, which in this case is the blue cluster represented by the center $C_{\alpha}^{1}$

${ }^{5}$ However, in contrast to the k-means algorithm, the proposed assignment rule is used in the eigenspace rather than in the original input space. 
As mentioned earlier, the initial clustering model is optimal in the sense that the kernel bandwidth and the number of clusters are carefully chosen by means of a rigorous model selection scheme. However, in order for the AKSC method to be a reliable fault detection tool, a calibration phase is crucial. More precisely, the calibration permits to automatically re-tune the parameters in order to minimize the false alarms and maximize the identification accuracy. The user only needs to specify the length of the calibration period $\mathrm{L}_{\mathrm{cal}}$ and the tolerance $\mathrm{THR}_{\text {new }}$ for the appearance of new clusters over time. Furthermore, to avoid the selection of a large bandwidth which may prevent the detection of failures, the minimum number of clusters is set to $k=2$.

\subsection{Automated fault detection}

In order for the AKSC algorithm to characterize the changing distribution of the data and to raise warnings in real time, two damage indicators are proposed. The first one, denoted by $\mathrm{DI}_{1}$, indicates the maximum similarity value between the current data point $\mathbf{x}_{t}$ and the actual cluster centroids:

$$
\mathrm{DI}_{1}\left(\mathbf{x}_{t}, \alpha_{t}^{(l)}\right)=\frac{1}{2}\left(\max _{C^{m}} K\left(\mathbf{x}_{t}, C^{m}\right)+\max _{C_{\alpha}^{m}} K_{\alpha}\left(\alpha_{t}^{(l)}, C_{\alpha}^{m}\right)\right)
$$

where $m=1, \ldots, k, K$ denotes the RBF kernel similarity function and $K_{\alpha}=\frac{\alpha^{(l)} \alpha^{(m)}}{\left\|\alpha^{(l)}\right\| \cdot\left\|\alpha^{(m)}\right\|}$ is the cosine similarity in terms of the eigenvectors of the (weighted and centered) kernel matrix given by eq. (3). Basically, the similarities in both the original input space and the eigenspace have been combined, with the purpose of making the detection scheme more robust against noise. This outlier indicator is then post-processed in order to be a monotonic non-increasing function (until the eventual detection of failure). Furthermore, $\mathrm{DI}_{1}$ is used by the $\mathrm{AKSC}$ algorithm to create a new cluster and raise an alarm about a possible failure.

The second outlier indicator is not directly employed for the automatic decision making, but acts more as an additional information which helps to make the whole algorithmic solution more reliable. Suppose that at the end of the calibration period a certain bandwidth of the RBF kernel $\sigma_{\text {cal }}$ has been selected. During the acquisition of new streaming data coming from the sensors, together with the cluster centers $C_{\alpha}^{1}, \ldots, C_{\alpha}^{k}$, also the bandwidth is updated as $\sigma_{t}=c_{\sigma} M_{1: t}$. Here the proportionality constant $c_{\sigma}$ has been tuned during the initialization period, and $M_{1: t}$ represents the median of the pairwise distances between the input data acquired from the beginning until the current time step $t$. This estimation of the bandwidth was suggested by [31] for time-series analysis. The second outlier indicator provided by the proposed AKSC method is defined as follows:

$$
\mathrm{DI}_{2}=\left|\frac{\sigma_{t}-\sigma_{\mathrm{cal}}}{\operatorname{std}\left(\left[\sigma_{1}, \ldots, \sigma_{t}\right]\right)}\right|
$$

where $\operatorname{std}(\cdot)$ indicates the standard deviation. Roughly speaking, $\mathrm{DI}_{2}$ measures the (standardized) difference between the data distribution at the end of the calibration period (where the structure is considered undamaged) and the current distribution. In case a major shift happens (i. e. $\mathrm{DI}_{2}>3$ ), the user is quickly notified. 
The non-stationary behavior of the data distribution can be modeled by the AKSC algorithm also by means of merging of existing clusters. However, this specific case does not trigger any alarm. In the proposed algorithm, two clusters (represented by their centroids) are merged if their similarity is greater than $\mathrm{THR}_{\mathrm{mrg}}$, which indicates a user-defined threshold.

The whole AKSC tool for real-time structural health monitoring is summarized in Algorithm 1. The related Matlab package can be downloaded from:

http: / / www.esat.kuleuven.be/stadius/ADB/langone/AKSClab.php

\section{Validation of the method}

In order to show the reader the working mechanism of the proposed fault detection strategy, first the simulation results related to a computer generated example are presented. Later on, the experimental outcomes concerning a unique dataset are presented. This dataset was obtained by monitoring a concrete bridge for almost a year before introducing realistic damage in a controlled way, and is referred to as the Z24 benchmark [32, 33, 34].

\subsection{Proof of concept on a simulated nonlinear system}

The synthetic example concerns a nonlinear system extensively used in the process monitoring literature and proposed originally in [35]. In particular, the system is described by three variables $y_{1}, y_{2}$ and $y_{3}$ that are different polynomial expressions of a random source variable $t$. The measured variables are corrupted by Gaussian noise variables $n_{1}, n_{2}$ and $n_{3}$, with variance equal to 0.01 (and zero mean). The variable $t$ is uniformly distributed between 0.01 and 2. Furthermore, two disturbances are introduced for the process variables $y_{1}$ and $y_{2}$ starting from sample 101:

- fault 1: a step bias of $y_{2}$ by -1 from the 101 st sample

- fault 2: a ramp change of $y_{1}$ by adding $0.03\left(s_{n}-100\right)$ from the 101 st sample, where $s_{n}$ indicates the sample number.

The related simulation model is described by the following set of equations $[36,37]$ :

$$
\begin{array}{lcc}
y_{1} & = & t+n_{1} \\
y_{2} & = & t^{2}-3 t+n_{2} \\
y_{3} & = & -t^{3}+3 t^{2}+n_{3} .
\end{array}
$$

The corresponding data distribution, characterized by 300 samples, is depicted in Figure 6. The figure shows that this system is nonlinear and that, since it is continuously shifting towards a faulty behaviour, it is difficult to identify the disturbances from normal operating data.

The results obtained using the AKSC approach are depicted in Figure 7. A total of 50 samples for the initialization, 25 samples for the calibration and the rest $(250$ samples) for testing have been used. Furthermore, only damage indicator $\mathrm{DI}_{1}$ is used for the decision making. The left side of the figure refers to setting a tolerance tol $\mathrm{l}_{\text {new }}=$ 1 and the right side relates to having tol ${ }_{\text {new }}=2$ in algorithm 1 . It can be noticed 


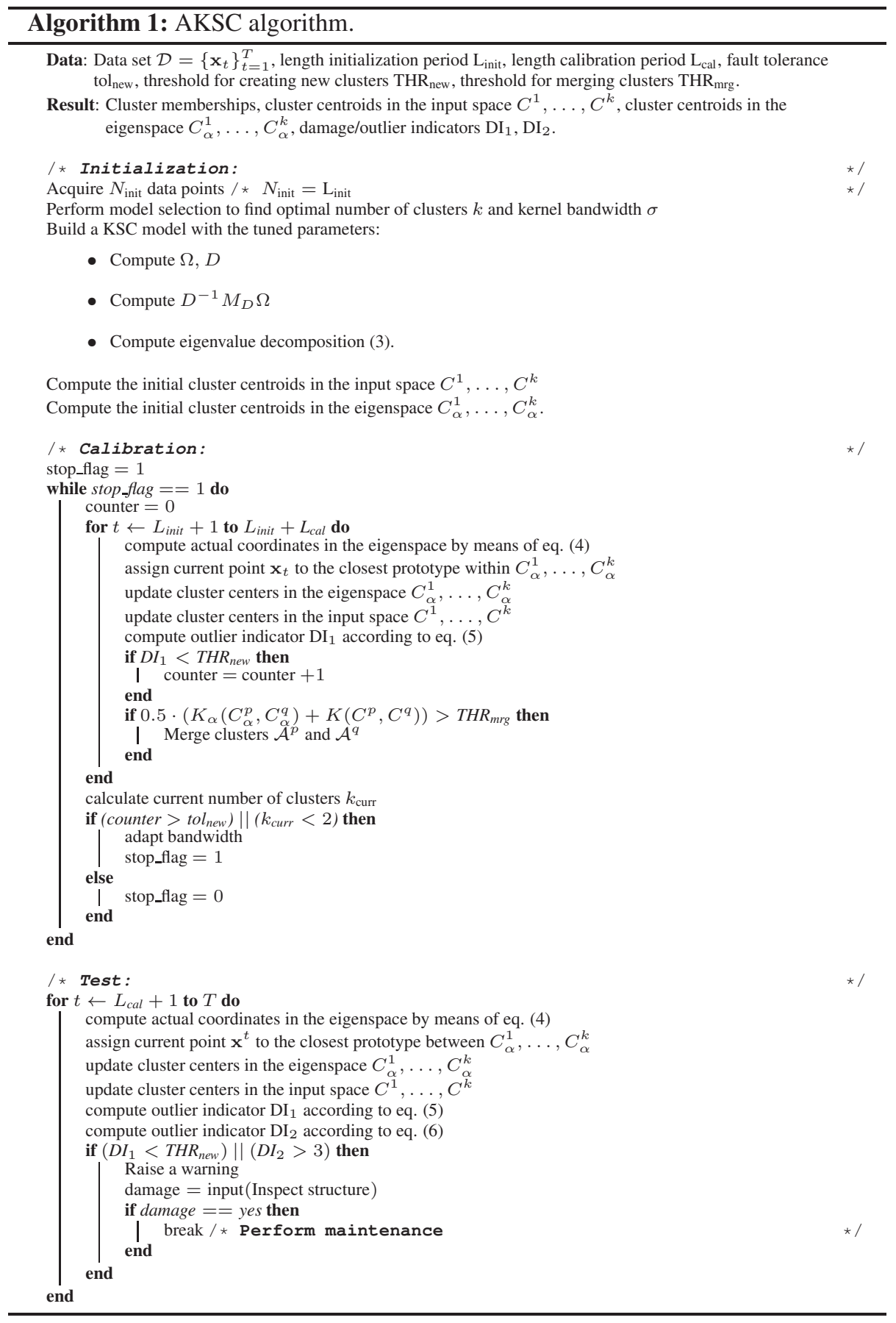




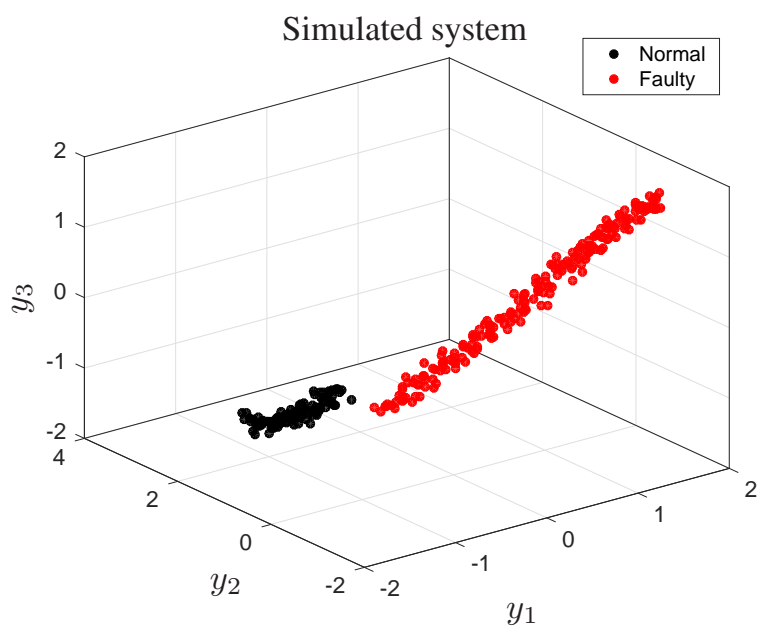

Figure 6: Synthetic dataset generated by means of eq. (7).

that in case of tol $\mathrm{l}_{\text {new }}=1$, the warning raised by the algorithm, detected by $\mathrm{DI}_{1}$, is given at time step 101 . The indicator $\mathrm{DI}_{2}$ suggests possible failures at time step 82 , thus in this case it would produce a false alarm if used for the decision making ${ }^{6}$. On the other hand, if the tolerance tol $\mathrm{l}_{\text {new }}=2$ is used, two alarms are raised at time steps 101 and 238. These outcomes show the effect of different values for the tolerance

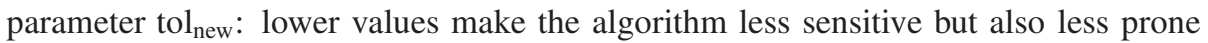
to false alarms, while higher values mean higher detection rate at the expenses of an increased chance for false alarms. However, in this specific example, the proposed approach is able to recognize the change of behavior in both cases, without producing any false alarm: in the first case the faulty regime is described by means of one cluster, in the second case it is modelled via the creation of 2 clusters.

\subsection{Experimental results on the Z24 bridge benchmark}

During the year before demolition, a long-term continuous monitoring of the Z24 bridge overpassing the A1 highway between Bern and Zurich took place. During the month before complete demolition, the bridge was gradually damaged in a controlled way, with the continuous monitoring system composed of 16 accelerometers still running. The Z24 bridge project was unique because it involved long-term continuous vibration monitoring of a full-scale structure, where at the end of the monitoring period, realistic damage was applied in a controlled way. The data have therefore been presented as a benchmark study for algorithms for structural health monitoring and fault detection [32, 33, 34].

From the recorded acceleration data four main eigen-modes, for which the natural frequencies could be identified with sufficient accuracy, were extracted. This resulted in a dataset [38] constituted by 5652 samples and 4 damage-sensitive features (i.e.

\footnotetext{
${ }^{6}$ However, by setting the control limit to 3.5 instead of 3 the false alarm would not be raised.
} 


$$
\text { tol }_{\text {new }}=1
$$

Initialization, calibration, testing

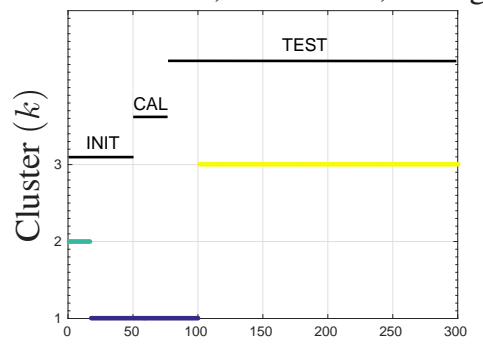

time idx
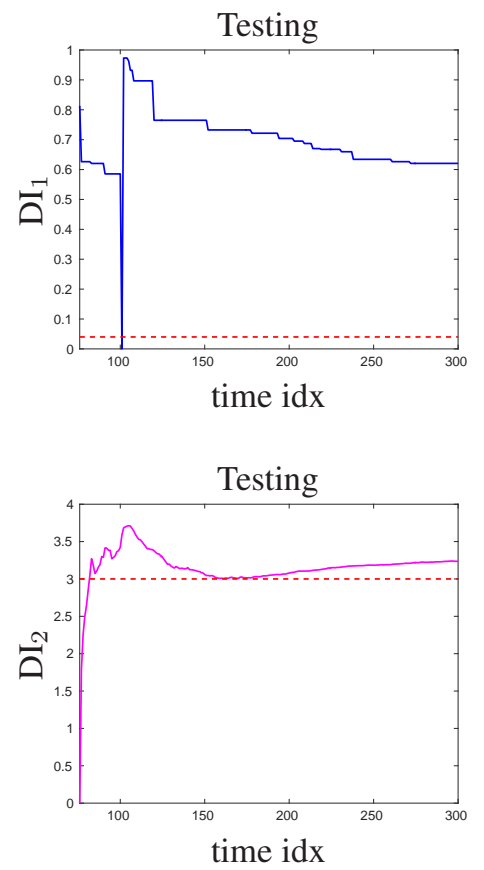

tol $_{\text {new }}=2$

Initialization, calibration, testing

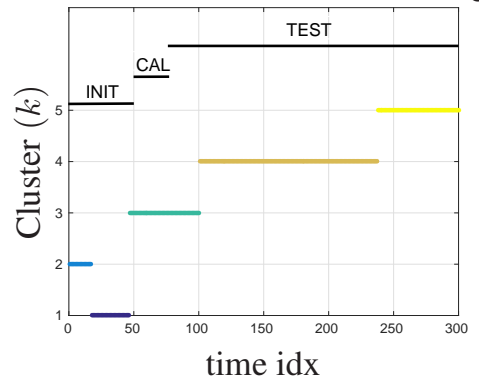

Testing
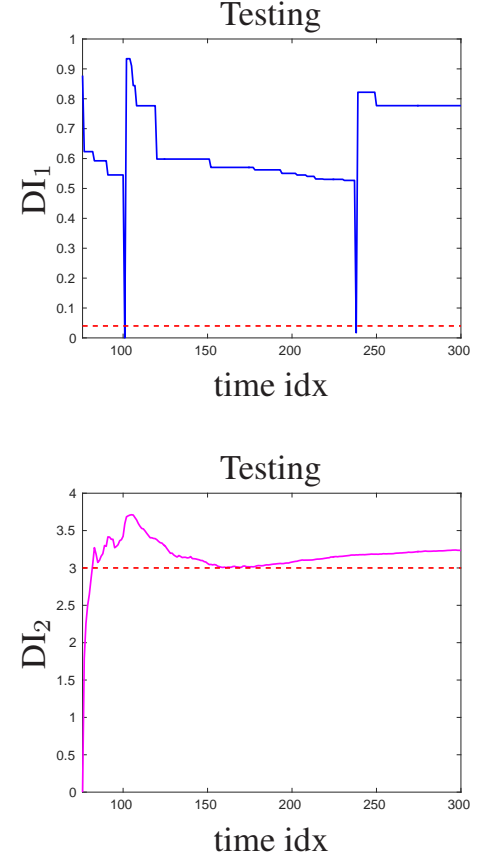

Figure 7: Results produced by AKSC on the synthetic dataset described by eq. (7). (Top) Regimes identified by the adaptive clustering algorithm. (Middle) First damage indicator, on which the decision making is based in these experiments: the control limit is 0.04. (Bottom) Second damage indicator with control limit equals to 3 . The first tolerance threshold allows to detect the faulty behavior starting from time step 101, while $\mathrm{DI}_{2}$ raises a false alarm at time step 82 (however, a control limit of 3.5 would avoid this false alarm). Notice that by setting tol $\mathrm{l}_{\text {new }}=1$ one cluster is created, while with tol $\mathrm{l}_{\text {new }}=2$ the faulty condition is described by means of 2 regimes created at time steps 101 and 238 . 


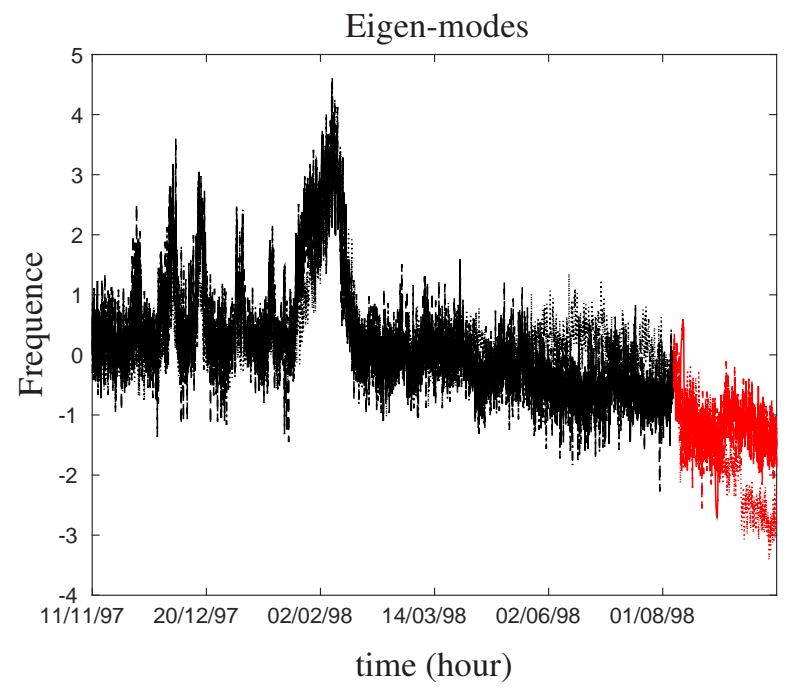

Figure 8: $\mathbf{Z 2 4}$ bridge benchmark dataset. The lines in red color refer to the period when the controlled damaging process started.

natural frequencies), which is illustrated in Figure 8 (where the 4 modes have been ormalized and plotted together).

The clustering outcomes produced by the proposed strategy are illustrated in Figure 9, where the following setting for the input variables of algorithm 1 have been used: tol $_{\text {new }}=1, \mathrm{THR}_{\text {new }}=0.01, \mathrm{THR}_{\mathrm{mrg}}=0.75$. This setting means that a point is considered to be an outlier if its similarity with all the existing clusters is less than $1 \%$, and two clusters are merged if their similarity is above $75 \%$. Furthermore, the left side of Figure 9 refers to training and calibrating using only one month of data $\left(\mathrm{L}_{\text {init }}=480\right.$, i. e. 20 days, $\mathrm{L}_{\mathrm{cal}}=240$, i. e. 10 days), while the right side shows the results obtained by using three months of data for initialization and calibration. From Figure 9 it can be noticed how the proposed method is able to detect the change in the structure due to the induced damage in both scenarios.

In the first scenario, i.e. $\mathrm{L}_{\text {init }}+\mathrm{L}_{\mathrm{cal}} \approx 1$ month (left side of Figure 9), the detection happens on August 7, 1998. This is in line with the detection time reported in [38]. In this work an autoregressive model with exogenous inputs (ARX) relating the temperature and the 4 natural frequencies was identified, and a damage was located when the simulated eigenfrequencies were deviating for a large extent from the measured ones, more precisely on August 15, 1998 in case of the first eigenmode and on August 7,8 for eigenfrequencies 2, 3, 4. Notice that a direct comparison between the proposed algorithm and the aforementioned ARX model is not appropriate, due to the fact that AKSC is an unsupervised learning method which does not make any assumption about the physical process underlying the structural behavior of the bridge, as the ARX model does. Still, AKSC allows a timely detection of the damage and with much less training datapoints. However, a false alarm is raised in the beginning when the structure is not damaged, at time step 1900 (i.e. February 3, 1998). We know that in this period (February 1998) the temperature was below zero degrees, and this probably caused the 
rapid increase of Young's modulus of the asphalt layer, resulting in a peak present in all of the vibration modes (see Figure 8). Thus, in view of this consideration the first warning raised by the AKSC method makes sense because it is related to a change in bridge dynamics, tough this change does not correspond to a structural failure.

In the second scenario, corresponding to $\mathrm{L}_{\mathrm{init}}+\mathrm{L}_{\mathrm{cal}} \approx 3$ months (right side of Figure 9), no false alarms are raised, but the detection of the structural damage in the end of the monitoring period is delayed. This is not surprising because after including the winter period in the training data, the estimated support of the normal behavior distribution gets enlarged, which in this case reduces the sensitivity of the clustering model to the structural changes.

Finally, for comparison purposes, in Figure 10 the results provided by the fuzzy Cmeans algorithm [39] are shown, which up to our knowledge is among the most used clustering techniques for structural health monitoring. In particular, we have run the method using the (default) Euclidean distance measure. The number of clusters has been set to $k=2$, and the first 480 samples have been used, as was done previously in case of the AKSC approach. Afterwards, every new point is assigned to the closest mean with a certain membership, whose maximum value is plotted in the Figure. It can be observed that the maximum membership never goes below the threshold, meaning that damage is not detected. This is probably due to the fact that fuzzy C-means, in contrast to the proposed technique, can only discover linearly separable clusters, which seems not adequate to model the bridge dynamics.

\section{Conclusions}

In this paper a novel approach for structural health monitoring has been introduced, which unifies the data normalization and damage detection steps. The proposed algorithm, called adaptive kernel spectral clustering (AKSC), is initialized and calibrated in a phase when the structure is undamaged. The calibration process consists of an online model selection process which allows to maximize the detection rate and minimize the number of false alarms. After the calibration, the algorithm adapts to changes in the data distribution by merging existing clusters or creating new clusters. Two different damage indicator variables are introduced, which permit the identification of suspicious structural behavior upon their occurrence. Finally, experimental results on a synthetic example and the Z24 concrete bridge benchmark have shown the benefit of the proposed strategy. Future work may consist of setting-up an adaptive semi-supervised clustering technique, which can exploit some form of prior knowledge to improve the fault detection strategy.

\section{Acknowledgment}

EU: The research leading to these results has received funding from the European Research Council under the European Union's Seventh Framework Programme (FP7/2007-2013) / ERC AdG A-DATADRIVE-B (290923). This paper reflects only the authors' views and the Union is not liable for any use that may be made of the contained information. Research Council KUL: CoE PFV/10/002 (OPTEC), BIL12/11T; PhD/Postdoc grants Flemish Government: FWO: projects: G.0377.12 (Structured systems), G.088114N (Tensor based data similarity); PhD/Postdoc grant iMinds Medical Information Technologies SBO 2015 IWT: POM II SBO 100031 Belgian Federal Science Policy Office: IUAP P7/19 (DYSCO, Dynamical systems, control and optimization, 2012-2017). All authors are members of OPTEC, and this research was partially supported by a Postdoctoral Fellowship from the Research Foundation - Flanders (FWO), Belgium, provided to E. Reynders. 

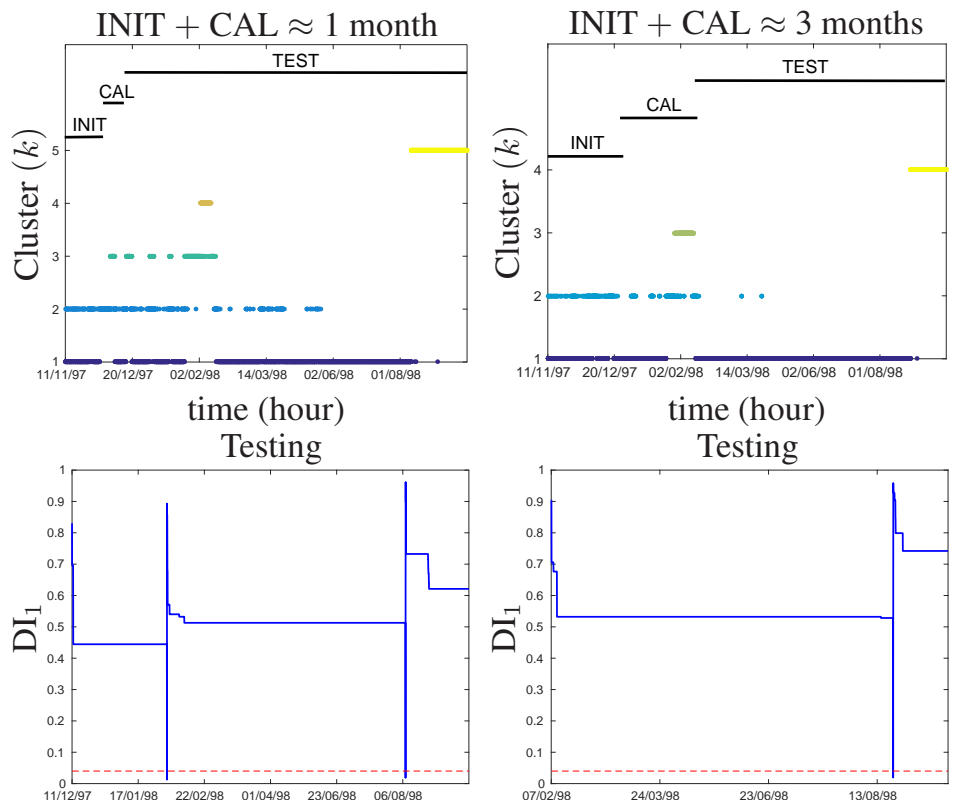

time (hour)

time (hour)

Testing

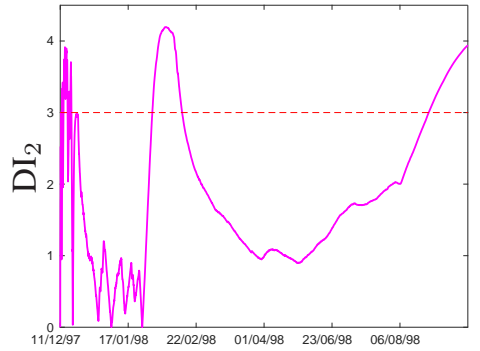

Testing

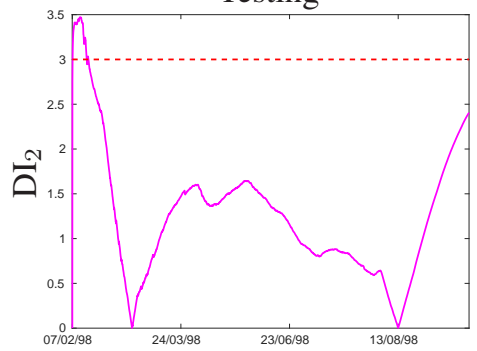

time (hour)

Figure 9: Z24 bridge results given by AKSC. (Top) Regimes identified by the adaptive clustering algorithm. (Middle) First damage indicator. (Bottom) Second damage indicator. Left $\mathrm{L}_{\text {init }}+\mathrm{L}_{\text {cal }} \approx 1$ month. Cluster number 4 is generated on February 3, 1998, and it is probably describing the rapid increase of Young's modulus of the asphalt layer due to low temperature. Cluster number 5 gets created around 3 days after the starting of the controlled damaging process (i.e. August 7, 1998), which is then detected by the AKSC algorithm. Right $\mathrm{L}_{\text {init }}+\mathrm{L}_{\text {cal }} \approx 3$ months. No false alarms are raised, but the damage condition is detected with more delay. 


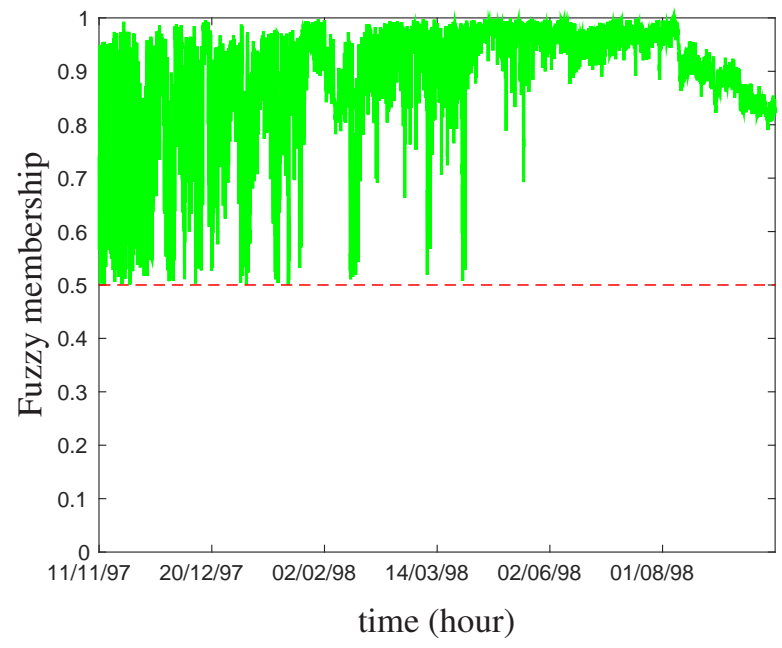

Figure 10: Z24 bridge results produced by fuzzy C-means. The maximum membership is used as damage or outlier indicator. Since the variable never takes values below the threshold, there is no detection of damage.

414

\section{References}

[1] C. R. Farrar, K. Worden, An introduction to structural health monitoring, Philosophical Transactions of the Royal Society of London A: Mathematical, Physical and Engineering Sciences 365 (1851) (2007) 303-315.

[2] S. Glaser, A. Tolman, Sense of sensing: From data to informed decisions for the built environment, Journal of Infrastructure Systems 14 (2008) 4-14.

[3] E. Reynders, System identification methods for (operational) modal analysis: Review and comparison, Archives of Computational Methods in Engineering 19 (1) (2012) 51-124.

[4] E. Reynders, G. Wursten, G. De Roeck, Output-only structural health monitoring in changing environmental conditions by means of nonlinear system identification, Structural Health Monitoring (SHM) 13 (1) (2014) 82-93.

[5] N. Dervilis, K. Worden, E. Cross, On robust regression analysis as a means of exploring environmental and operational conditions for SHM data, Journal of Sound and Vibration 347 (2015) 279 - 296.

[6] J. Kullaa, Distinguishing between sensor fault, structural damage, and environmental or operational effects in structural health monitoring, Mechanical Systems and Signal Processing 25 (8) (2011) 2976 - 2989.

[7] M. D. Spiridonakos, E. N. Chatzi, B. Sudre, Polynomial chaos expansion models for the monitoring of structures under operational variability, ASCE-ASME Journal of Risk and Uncertainty in Engineering Systems Part A: Civil Engineering (B) (2016) 4016003.

[8] K. Worden, G. Manson, N. R. J. Fieller, Damage detection using outlier analysis, Journal of Sound and Vibration 229 (3) (2000) 647-667.

[9] H. Sohn, K. Worden, C. R. Farrar, Statistical damage classification under changing environmental and operational conditions, SJournal of intelligent material systems and structures 13 (9) (2002) 561-574.

[10] E. Figueiredo, G. Park, C. R. Farrar, K. Worden, J. Figueiras, Machine learning algorithms for damage detection under operational and environmental variability, Structural Health Monitoring (SHM) 10 (6) (2011) 559-572.

[11] A. Abdul-Aziz, M. R. Woike, N. C. Oza, B. L. Matthews, J. D. Lekki, Rotor health monitoring combining spin tests and data-driven anomaly detection methods, Structural Health Monitoring (SHM) 11 (1) (2012) 3-12.

[12] A. Deraemaeker, E. Reynders, G. De Roeck, J. Kullaa, Vibration-based structural health monitoring using output-only measurements under changing environment, Mechanical Systems and Signal Processing 22 (1) (2008) 34 - 56. 
[13] F. Magalhaes, A. Cunha, E. Caetano, Vibration based structural health monitoring of an arch bridge: From automated to damage detection, Mechanical Systems and Signal Processing 28 (0) (2012) 212 - 228.

[14] A. K. Jain, Data clustering: 50 years beyond k-means, Pattern Recogn. Lett. 31 (8) (2010) 651-666.

[15] H. Sohn, S. D. Kim, K. Harries, Reference-free damage classification based on cluster analysis, Computer-Aided Civil and Infrastructure Engineering 23 (5) (2008) 324-338.

[16] K. N. Kesavan, A. S. Kiremidjian, A wavelet-based damage diagnosis algorithm using principal component analysis, Structural Control and Health Monitoring 19 (8) (2012) 672-685.

[17] L. Palomino, J. Steffen, Valder, R. Finzi, Fuzzy cluster analysis methods applied to impedance based structural health monitoring for damage classification, in: R. Allemang, J. De Clerck, C. Niezrecki, J. Blough (Eds.), Topics in Modal Analysis II, Volume 6, Conference Proceedings of the Society for Experimental Mechanics Series, Springer Berlin Heidelberg, 2012, pp. 205-212.

[18] G. Park, H. Cudney, D. Inman, Impedance-based health monitoring of civil structural components, J. Infrastruct. Syst. 4 (6) (2000) 153160.

[19] A. Amadore, G. Bosurgi, O. Pellegrino, Classification of measures from deflection tests by means of fuzzy clustering techniques, Construction and Building Materials 53 (0) (2014) 173 - 181.

[20] J. Santos, L. Calado, A. Orcesi, C. Cremona, Adaptive detection of structural changes based on unsupervised learning and moving time-windows, in: EWSHM - 7th European Workshop on Structural Health Monitoring, 2014.

[21] A. Curi, Application of symbolic data analysis for structural modification assessment, Engineering Structures 32 (3) (2010) $762-775$.

[22] F. R. K. Chung, Spectral Graph Theory, American Mathematical Society, 1997.

[23] A. Y. Ng, M. I. Jordan, Y. Weiss, On spectral clustering: Analysis and an algorithm, in: T. G. Dietterich, S. Becker, Z. Ghahramani (Eds.), Advances in Neural Information Processing Systems 14, MIT Press, Cambridge, MA, 2002, pp. 849-856.

[24] U. von Luxburg, A tutorial on spectral clustering, Statistics and Computing 17 (4) (2007) 395-416.

[25] C. Alzate, J. A. K. Suykens, Multiway spectral clustering with out-of-sample extensions through weighted kernel PCA, IEEE Transactions on Pattern Analysis and Machine Intelligence 32 (2) (2010) 335-347.

[26] R. Langone, R. Mall, J. A. K. Suykens, Soft kernel spectral clustering., in: Proc. of the International Joint Conference on Neural Networks (IJCNN 2013), 2013, pp. $1-8$.

[27] R. Langone, O. M. Agudelo, B. De Moor, J. A. K. Suykens, Incremental kernel spectral clustering for online learning of non-stationary data, Neurocomputing 139 (0) (2014) 246-260.

[28] J. Shawe-Taylor, N. Cristianini, Kernel Methods for Pattern Analysis, Cambridge University Press, 2004.

[29] S. Mika, B. Schölkopf, A. J. Smola, K. R. Müller, M. Scholz, G. Rätsch, Kernel PCA and de-noising in feature spaces, in: M. S. Kearns, S. A. Solla, D. A. Cohn (Eds.), Advances in Neural Information Processing Systems 11, MIT Press, 1999.

[30] C. Alzate, J. A. K. Suykens, Out-of-sample eigenvectors in kernel spectral clustering, in: Proc. of the International Joint Conference on Neural Networks (IJCNN 2011), 2011, pp. 2349-2356.

[31] M. Cuturi, Fast global alignment kernels, in: Proceedings of the 28th International Conference on Machine Learning, ICML 2011, Bellevue, Washington, USA, June 28 - July 2, 2011, 2011, pp. 929-936.

[32] J. Maeck, G. De Roeck, Description of Z24 benchmark, Mechanical Systems and Signal Processing 17 (1) (2003) 127 $-131$.

[33] E. Reynders, G. De Roeck, Continuous Vibration Monitoring and Progressive Damage Testing on the Z24 Bridge, John Wiley \& Sons, Ltd, New York, NY, 2009, pp. 2149-2158.

[34] E. Reynders, G. De Roeck, Vibration-based damage identification: The Z24 bridge benchmark, in: Encyclopedia of Earthquake Engineering, Springer Berlin Heidelberg, 2015, pp. 1-8.

[35] D. Dong, T. McAvoy, Nonlinear principal component analysis based on principal curves and neural networks, Computers and Chemical Engineering 20 (1) (1996) 65 - 78. 
[36] J.-H. Cho, J.-M. Lee, S. W. Choi, D. Lee, I.-B. Lee, Fault identification for process monitoring using kernel principal component analysis, Chemical Engineering Science 60 (1) (2005) 279 - 288.

48 [37] Z. Li, U. Kruger, L. Xie, A. Almansoori, H. Su, Adaptive KPCA modeling of nonlinear systems, Signal Processing, IEEE Transactions on 63 (9) (2015) 2364-2376.

490 [38] B. Peeters, G. De Roeck, One-year monitoring of the z24-bridge: environmental effects versus damage events, Earth491 quake Engineering \& Structural Dynamics 30 (2) (2001) 149-171.

492 [39] J. Bezdek, R. Ehrlich, W. Full, FCM: The fuzzy C-means clustering algorithm, Computers \& Geosciences 10 (2-3) 493 (1984) 191-203. 